\title{
33
}

\section{Yes, we can clear this: a tissue clearing toolbox for every organ}

Monika Pawłowska $^{1,2}$, Paweł Matryba ${ }^{3,4}$, Anna Połeć 5 , Marzena Stefaniuk ${ }^{1}$

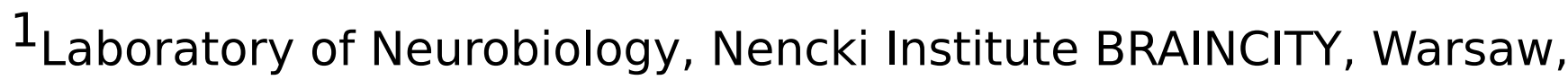
Poland. ${ }^{2}$ Faculty of Physics, University of Warsaw, Poland.

${ }^{3}$ Department of Immunology, Medical University of Warsaw, Poland. ${ }^{4}$ The Doctoral School of the Medical University of Warsaw, Medical University of Warsaw, Poland. ${ }^{5}$ Oslo University Hospital, Norway

\section{Abstract Text}

Within less than a decade since the modern tissue optical clearing (TOC) techniques were presented, approximately 25 original methods have been published along with more than a hundred of their modifications. Such rapid growth of the field, however, is not only valuable, but can also be perceived as confusing. Instead of introducing yet another new method, here we present examples of TOC protocols best suited to different applications that were screened and verified by our laboratory over the course of the last few years. Based on the conducted research, we propose different strategies to be applied for a variety of samples, ranging from millimeter-sized mouse lymph nodes, through centimeter sized rodent brains, muscles and internal organs, up to the entire mouse body imaged in a self-build light-sheet imaging system designed specifically for this purpose [Bożycki 2018]. We also include images from centimeter-sized human formalin-fixed paraffin-embedded colorectal carcinoma tissue.

Based on this, we propose guidelines on how to choose the most suitable clearing method depending on sample size, composition and desired staining. For instance, some tissues, such as skeletal muscles, heart, and liver, require prior decolorization best achieved by CUBIC-derived methods. On the other hand, solvent-based 
methods with their higher refractive index and stronger delipidation tend to yield higher transparency.

Finally, we present that the understanding of the mechanism that lies behind each category of TOC method can be used both for appropriate selection of an existing protocol, but more importantly, for creating a unique approach that suits the needs of your research. For example, although neither CUBIC, nor FDISCO, could lead us to successful imaging of the entire murine penis, proper combination of these allowed us to obtain both optimal immunostaining and adequate transparency to get the cellular-resolution view on this specimen for the first time [Matryba 2020]. 